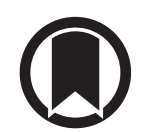

CrossMark

\title{
Obstructive sleep apnoea severity and liver steatosis measured by magnetic resonance imaging
}

\begin{abstract}
To the Editor:
Obstructive sleep apnoea (OSA) and non-alcoholic fatty liver disease (NAFLD) are two of many diseases associated with obesity. NAFLD is a common condition ranging in severity from liver steatosis to non-alcoholic steatohepatitis and liver fibrosis, the last step of NAFLD progression. Numerous studies have investigated whether the frequent co-occurrence of OSA and NAFLD simply reflects their link to obesity, or whether there is an independent pathophysiological interconnection between the two diseases (see [1] for comprehensive review). In animal models, intermittent hypoxia mimicking OSA has been shown to cause insulin resistance, dysfunction of key steps in hepatic lipid metabolism, liver steatosis and fibrosis [1]. In the clinical setting, the increased burden of chronic liver disease and the practical limitations of using liver biopsy has led to the development of serum- or imaging-based non-invasive tests, allowing reliable detection and quantification of liver steatosis and fibrosis [2]. Our group has recently reported that severe OSA is independently associated with increased liver stiffness, a validated marker of liver fibrosis, in patients with metabolic comorbidities [3]. In the same clinic-based cohort, we aimed to determine whether OSA severity is also independently associated with liver steatosis, the first step of NAFLD. We used magnetic resonance imaging (MRI), which is considered as the gold standard for non-invasive assessment of liver steatosis according to current European guidelines [4].
\end{abstract}

As previously described [3], the study was nested within the NUMEVOX cohort (Clinicaltrials.gov NCT00997165), a clinic-based cohort including patients with at least one criterion for metabolic syndrome referred to Angers University Hospital (Angers, France). Exclusion criteria included age $<18$ or $>65$ years, causes of chronic liver disease other than NAFLD, complicated cirrhosis and insulin-treated diabetes. Baseline evaluation included anthropometric data, medical history, medication use and fasting blood tests including blood glucose, insulin, liver enzymes and total bilirubin. All patients fulfilled the Berlin questionnaire to evaluate the clinical likelihood of OSA.

Between March 2008 and August 2017, 205 patients subsequently investigated by overnight polysomnography (PSG) based on a positive Berlin questionnaire were eligible for the present study. Overnight PSGs (CID 102; Cidelec, Angers, France) were performed (date of MRI \pm 1 month) and manually scored according to standard criteria [5]. Patients were examined on a 1.5T SIGNA HDx MRI (GE Healthcare, Milwaukee, WI, USA) upgraded to HDxt in 2011. As previously described [6], the protocol systematically included a breath-hold multi-echo gradient echo sequence comprising alternating in-phase and opposed-phase echoes with 10 echoes. The sequence was performed with a body coil, using the following acquisition parameters: matrix $240 \times 192$; field of vision $470 \times 376$; slice thickness $10 \mathrm{~mm}$; 1 excitation; bandwidth $488 \mathrm{kHz}$. A $20^{\circ}$ flip angle was used in order to avoid the T1 effect. MRI measurement of liver proton density fat-fraction (PDFF-MRI) was performed with MRQuantif free software developed in the University of Rennes - France (imagemed.univ-rennes1.fr/en/mrquantif/ download.php) using the two-point Dixon method corrected by $\mathrm{T}^{*}$. Three regions of interest were placed in the right lobe of the liver on a MRI slice with a large volume of right lobe, avoiding large vascular structures, bile ducts, liver lesions or artefacts, and one region of interest was placed in the air

@ERSpublications

In patients with OSA and metabolic comorbidities, liver steatosis measured by magnetic resonance imaging is associated with male sex and insulin resistance, but not with OSA severity and nocturnal hypoxia http://bit.ly/2kqyi3O

Cite this article as: Trzepizur W, Boursier J, Berréhare A, et al. Obstructive sleep apnoea severity and liver steatosis measured by magnetic resonance imaging. Eur Respir J 2020; 55: 1901514 [https://doi.org/ 10.1183/13993003.01514-2019]. 
outside of the body for noise measurement. The presence of liver steatosis was defined by a PDFF-MRI value $>5.6 \%[4]$.

Out of 205 eligible patients, 38 were excluded from the analysis due to unavailability or contraindications to MRI. Our final sample size therefore comprised 167 patients who were predominantly male $(65 \%)$, obese or overweight (mean \pm SD body mass index (BMI) $30.3 \pm 4.9 \mathrm{~kg} \cdot \mathrm{m}^{-2}$ ), frequently presenting metabolic comorbidities (diabetes 14.5\%; metabolic syndrome 52\%). OSA was confirmed by PSG in all patients, with $94(56.3 \%)$ mild-to-moderate and 73 (44\%) severe OSA. Liver steatosis was diagnosed in 85 (51\%) patients on MRI. The association of OSA severity, indices of nocturnal hypoxaemia and covariates with the presence of liver steatosis was assessed by logistic regression analysis. On univariate analysis, severe OSA was associated with a significantly increased risk of liver steatosis (OR 1.88, 95\% CI 1.01-3.51; p=0.0473) (table 1). On multivariate analysis, liver steatosis was associated with male sex (OR 2.69, 95\% CI 1.23-5.87; $\mathrm{p}=0.0128$ ) and the homeostasis model assessment of insulin resistance (OR 2.06, 95\% CI 1.26-3.37 for $1 \mathrm{SD} ; \mathrm{p}=0.0039$ ), but not with OSA severity. There was no significant association between liver steatosis and hypoxaemia during sleep, as reflected by the oxygen desaturation index (ODI) and the percentage of sleep time with oxygen saturation $<90 \%\left(t_{90}\right)$. To avoid bias in choosing arbitrary cut-points, regression analyses were performed with OSA severity indices and PDFF-MRI values kept as continuous variables. On univariate analysis, PDFF-MRI was associated with apnoea-hypopnoea index $(p=0.0085)$, ODI $(p=0.0062)$ and $t_{90}(\mathrm{p}=0.0014)$, but the association was no longer significant after adjustment for confounders.

To our knowledge, this is the first study evaluating the relationship between OSA severity and liver steatosis measured by MRI. We found that severe OSA was associated with a significant increased risk of liver steatosis in patients with metabolic comorbidities. However, the association was no longer significant after adjustment for confounders.

The potential impact of OSA on liver fat accumulation has been studied in animal models. Lean and obese mice submitted to intermittent hypoxia, a hallmark of OSA, showed increased liver triglycerides content resulting in mild histological liver steatosis [7, 8]. Inconsistent results have been reported concerning the association between OSA and liver steatosis in clinical cohorts. Several studies performed in predominantly female morbidly obese patients undergoing intraoperative needle liver biopsy during bariatric surgery found no independent relationship after adjustment for confounders $[9,10]$. In contrast, clinical studies evaluating liver steatosis by abdominal ultrasonography suggested that OSA may modulate the early NAFLD phenotype $[11,12]$. In 175 non-obese individuals undergoing PSG and abdominal ultrasonography, QI et al. [11] found that the lowest oxygen saturation during sleep independently predicted the development of NAFLD in multi-regression analysis. Likewise, in a population including both lean and overweight NAFLD patients diagnosed using ultrasound, the severity of intermittent hypoxia was an independent predictor of increased liver enzymes [13]. However, a comprehensive review and meta-analysis have demonstrated that the overall performance of abdominal ultrasonography is clearly lower than that of MRI for the evaluation of hepatic steatosis [14]. Recent large clinic-based cohort studies demonstrated an independent association between OSA severity and liver steatosis assessed by clinical prediction rule combining risk factors and blood-based biomarkers $[15,16]$. However, the fact that the BMI is included in the calculation of these predictive scores must be taken into account as a potential limitation when interpreting these findings.

TABLE 1 Unadjusted and adjusted odds ratios for having liver steatosis on magnetic resonance imaging, according to obstructive sleep apnoea (OSA) severity, indices of hypoxaemia during sleep and covariates

\begin{tabular}{|c|c|c|c|c|}
\hline & Unadjusted OR (95\% CI) & p-value & Adjusted OR $(95 \% \mathrm{CI})$ & p-value \\
\hline Age 10 years & $0.92(0.68-1.25)$ & 0.6014 & & \\
\hline Male sex & $2.47(1.29-4.72)$ & 0.0064 & $2.69(1.23-5.87)$ & 0.0128 \\
\hline Body mass index $1 \mathrm{SD}$ & $1.72(1.22-2.42)$ & 0.0020 & $1.50(0.97-2.31)$ & 0.0656 \\
\hline Diabetes & $3.97(1.51-10.42)$ & 0.0051 & $2.44(0.81-7.35)$ & 0.1135 \\
\hline Hypertension & $1.77(0.96-3.24)$ & 0.0664 & & \\
\hline HOMA-IR" 1 SD & $2.89(1.79-4.68)$ & $<0.0001$ & $2.06(1.26-3.37)$ & 0.0039 \\
\hline Severe OSA & $1.88(1.01-3.51)$ & 0.0473 & $1.08(0.51-2.30)$ & 0.8400 \\
\hline ODI $\geqslant 20$ versus $<20$ events $\cdot \mathrm{h}^{-1}$ & $1.55(0.84-2.88)$ & 0.1607 & & \\
\hline$t_{90} \geqslant 2 \%$ versus $<2 \%$ & $1.85(0.99-3.44)$ & 0.0542 & & \\
\hline
\end{tabular}

HOMA-IR: homeostasis model assessment of insulin resistance; ODI: oxygen desaturation index; t90: sleep time with oxygen saturation $<90 \%$. \#: the HOMA-IR was calculated from fasting glucose and insulin concentrations, as follows: insulin $\left(\mathrm{mlU} \cdot \mathrm{L}^{-1}\right) \times \mathrm{glucose}\left(\mathrm{mmol} \cdot \mathrm{L}^{-1}\right) / 22.5$. 
A major strength of the present study is the use of gold-standard procedures for both OSA diagnosis (PSG) and non-invasive liver steatosis measurement (MRI) in a relatively large sample of patients. Altogether, the present results and those from bariatric surgery cohorts suggest that OSA might play a limited role in the development of liver steatosis $[9,10]$. Considering the limited impact of positive airway pressure (PAP) therapy alone on insulin resistance in obese patients with OSA, combined modality therapy including weight-loss interventions is a promising strategy for the management of patients with OSA, metabolic comorbidities and liver steatosis [17].

Conversely, recent clinical data suggested that OSA and its hypoxia-related consequences may accentuate liver injury in NAFLD, leading to a shift in the phenotype toward non-alcoholic steatohepatitis (NASH) and liver fibrosis. A recent cross-sectional study using a large national database demonstrated that patients with OSA are three times more likely to have NASH compared to non-OSA patients after controlling for metabolic comorbidities [18]. Within the NUMEVOX cohort, our group has recently reported that severe OSA is independently associated with increased liver stiffness, which may predispose to a higher risk of significant liver disease and poorer prognosis [3]. Further large prospective cohort studies using validated non-invasive tests should evaluate the nature of the relationship between OSA, liver steatosis, NASH and liver fibrosis. In addition, well-designed randomised controlled trials are required to evaluate the effect of PAP therapy, alone or combined with lifestyle interventions on NAFLD progression [19].

In conclusion, among patients with metabolic comorbidities, liver steatosis assessed by MRI was associated with male sex and insulin resistance, but not with OSA severity and nocturnal hypoxaemia. This study does not support the hypothesis of an independent contribution of OSA and its hypoxia-related consequences to liver fat accumulation.

Wojciech Trzepizur ${ }^{1,2}$, Jérôme Boursier ${ }^{3,4}$, Anna Berréhare ${ }^{1}$, Marc Le Vaillant ${ }^{5}$, Ramaroson Andriantsitohaina ${ }^{2}$, Pierre-Henri Ducluzeau, Séverine Dubois ${ }^{2,7}$, Samir Henni ${ }^{8,9}$, Pierre Abraham $\oplus^{8,9}$, Paul Calès ${ }^{3,4}$, Christophe Aubé ${ }^{4,10}$, Anita Paisant ${ }^{4,10}$ and Frédéric Gagnadoux ${ }^{1,2}$, on the behalf of the METABOL group ${ }^{11}$

${ }^{1}$ Département de Pneumologie et Médecine du Sommeil, Centre Hospitalier Universitaire, Angers, France. ${ }^{2}$ INSERM UMR 1063 SOPAM, Université d'Angers, Angers, France. ${ }^{3}$ Département d'Hépato-Gastroentérologie, Centre Hospitalier Universitaire, Angers, France. ${ }^{4}$ HIFIH, EA3859, Université d'Angers, Angers, France. ${ }^{5}$ Institut de Recherche en Santé Respiratoire des Pays de la Loire, Beaucouzé, France. ${ }^{6}$ Unité d'Endocrinologie-Diabétologie-Nutrition, Pole de Médecine, Centre Hospitalier Universitaire, Tours, France. ${ }^{7}$ Département d’Endocrinologie, Diabétologie et Nutrition, Centre Hospitalier Universitaire, Angers, France. ${ }^{8}$ Service de Médecine Vasculaire, Centre Hospitalier Universitaire, Angers, France. ${ }^{9}$ Institut MITOVASC, UMR CNRS 6015, INSERM 1083, Université d'Angers, Angers, France. ${ }^{10}$ Département de Radiologie, Centre Hospitalier Universitaire, Angers, France. ${ }^{11} \mathrm{~A}$ list of members of the METABOL group can be found in the Acknowledgements section.

Correspondence: Wojciech Trzepizur, Département de Pneumologie et Médecine du Sommeil, CHU d'Angers, 4 rue Larrey, 49100, Angers, France. E-mail: wotrzepizur@chu-angers.fr

Received: 30 July 2019 | Accepted after revision: 11 Sept 2019

Acknowledgements: The METABOL Group: Département de Pneumologie, CHU, Angers, France: Nicole Meslier, Pascaline Priou; Service d'Hépato-gastroentérologie, CHU, Angers, France: Frédéric Oberti, Isabelle Fouchard-Hubert, Adrien Lannes, Sandra Girres; Département d'Endocrinologie, Diabétologie, Nutrition, CHU, Angers, France: Ingrid Allix; Département de Médecine du Sport et Explorations Fonctionnelles Vasculaires, CHU, Angers, France: Georges Leftheriotis; INSERM UMR 1063 "SOPAM", Univeristé d'Angers, Angers, France: Carmen Martinez, Soazig Le Lay, Raffaella Soleti, Luisa Vergori; Département de la Recherche Clinique et Innovation, CHU, Angers, France: Jean-Marie Chrétien; Centre de Resource Biologique, CHU, Angers, France: Odile Blanchet, Belaid Sekour; Laboratoire HIFIH, EA3859, Université d'Angers, France: Gilles Hunault.

Conflict of interest: None declared.

\section{References}

1 Mesarwi OA, Loomba R, Malhotra A. Obstructive sleep apnea, hypoxia, and nonalcoholic fatty liver disease. Am J Respir Crit Care Med 2019; 199: 830-841.

2 Asrani SK. Incorporation of noninvasive measures of liver fibrosis into clinical practice: diagnosis and prognosis. Clin Gastroenterol Hepatol 2015; 13: 2190-2204.

3 Trzepizur W, Boursier J, Le Vaillant M, et al. Increased liver stiffness in patients with severe sleep apnoea and metabolic comorbidities. Eur Respir J 2018; 51: 1800601.

4 European Association for the Study of the Liver (EASL), European Association for the Study of Diabetes (EASD), European Association for the Study of Obesity (EASO). EASL-EASD-EASO clinical practice guidelines for the management of non-alcoholic fatty liver disease. J Hepatol 2016; 64: 1388-1402.

5 Berry RB, Budhiraja R, Gottlieb DJ, et al. Rules for scoring respiratory events in sleep: update of the 2007 AASM Manual for the Scoring of Sleep and Associated Events. Deliberations of the Sleep Apnea Definitions Task Force of the American Academy of Sleep Medicine. J Clin Sleep Med 2012; 8: 597-619.

6 Cesbron-Métivier E, Roullier V, Boursier J, et al. Noninvasive liver steatosis quantification using MRI techniques combined with blood markers. Eur J Gastroenterol Hepatol 2010; 22: 973-982. 
7 Li J, Grigoryev DN, Ye SQ, et al. Chronic intermittent hypoxia upregulates genes of lipid biosynthesis in obese mice. J Appl Physiol 2005; 99: 1643-1648.

8 Savransky V, Bevans S, Nanayakkara A, et al. Chronic intermittent hypoxia causes hepatitis in a mouse model of diet-induced fatty liver. Am J Physiol Gastrointest Liver Physiol 2007; 293: G871-G877.

9 Daltro C, Cotrim HP, Alves E, et al. Nonalcoholic fatty liver disease associated with obstructive sleep apnea: just a coincidence? Obes Surg 2010; 20: 1536-1543.

10 Aron-Wisnewsky J, Minville C, Tordjman J, et al. Chronic intermittent hypoxia is a major trigger for non-alcoholic fatty liver disease in morbid obese. J Hepatol 2012; 56: 225-233.

11 Qi J-C, Huang J-C, Lin Q-C, et al. Relationship between obstructive sleep apnea and nonalcoholic fatty liver disease in nonobese adults. Sleep Breath 2016; 20: 529-535.

12 Türkay C, Ozol D, Kasapoğlu B, et al. Influence of obstructive sleep apnea on fatty liver disease: role of chronic intermittent hypoxia. Respir Care 2012; 57: 244-249.

13 Lin Q-C, Chen L-D, Chen G-P, et al. Association between nocturnal hypoxia and liver injury in the setting of nonalcoholic fatty liver disease. Sleep Breath 2015; 19: 273-280.

14 Bohte AE, van Werven JR, Bipat S, et al. The diagnostic accuracy of US, CT, MRI and ${ }^{1} \mathrm{H}-\mathrm{MRS}$ for the evaluation of hepatic steatosis compared with liver biopsy: a meta-analysis. Eur Radiol 2011; 21: 87-97.

15 Minville C, Hilleret M-N, Tamisier R, et al. Nonalcoholic fatty liver disease, nocturnal hypoxia, and endothelial function in patients with sleep apnea. Chest 2014; 145: 525-533.

16 Trzepizur W, Boursier J, Mansour Y, et al. Association between severity of obstructive sleep apnea and blood markers of liver injury. Clin Gastroenterol Hepatol 2016; 14: 1657-1661.

17 Chirinos JA, Gurubhagavatula I, Teff K, et al. CPAP, weight loss, or both for obstructive sleep apnea. $N$ Engl J Med 2014; 370: 2265-2275.

18 Asfari MM, Niyazi F, Lopez R, et al. The association of nonalcoholic steatohepatitis and obstructive sleep apnea. Eur J Gastroenterol Hepatol 2017; 29: 1380-1384.

19 Labarca G, Cruz R, Jorquera J. Continuous positive airway pressure in patients with obstructive sleep apnea and non-alcoholic steatohepatitis: a systematic review and meta-analysis. J Clin Sleep Med 2018; 14: 133-139.

Copyright (c)ERS 2020 